\title{
Extensor Pollicis Longus Rupture after Mini TightRope Suspensionplasty
}

\author{
Mani Seetharaman, MD, MS ${ }^{1} \quad$ Mark A. Vitale, MD, MPH${ }^{1} \quad$ Kapil Desai, MD ${ }^{2} \quad$ John F. Crowe, MD \\ 1 ONS Foundation for Clinical Research and Education, ONS, PC, \\ Greenwich, Connecticut \\ 2 Department of Radiology, Greenwich Hospital, Greenwich, \\ Address for correspondence Mark A. Vitale, MD, MPH, Orthopaedic \\ and Neurosurgery Specialists (ONS), PC, 6 Greenwich Office Park, 40 \\ Valley Drive Greenwich, CT 06831 (e-mail: markavitale@yahoo.com).
} Connecticut

J Wrist Surg 2016;5:143-146

\begin{abstract}
Keywords

- EPL rupture

- Mini TightRope

- suspensionplasty

- trapeziectomy

Background Arthritis of the first carpometacarpal (CMC) joint has been surgically treated in multiple ways with varying levels of success as measured by subjective and objective measures. Trapeziectomy with numerous variations in suspensionplasty comprises one of the more commonly used surgical procedures. Recently, the Mini TightRope apparatus has been utilized as a new method for achieving suspensionplasty, and as such lacks significant review of use and safety in the literature.

Case Description An extensor pollicis longus (EPL) rupture following a trapeziectomy and Mini TightRope suspensionplasty for CMC arthritis of the thumb is presented. The patient successfully underwent an extensor indicis proprius (EIP) to EPL transfer to treat this complication.

Literature Review There is well-established documentation of injury to the extensor tendons from orthopedic hardware such as volar locking plates. Regarding use of the Mini TightRope apparatus, guidelines for placement of the suture button include caution to place the button away from the EPL tendon to minimize the chance of tendon irritation. Additionally, FiberWire sutures, a component of the apparatus, have been shown to demonstrate soft tissue reactions with adjacent inflammatory response. Published reports on adverse events utilizing this device have been limited to case reports including an index metacarpal fracture.

Clinical Relevance The aim of this case report was to cite an occurrence of EPL rupture following its use and discuss the possibilities of its direct contribution.
\end{abstract}

Symptomatic degenerative joint disease of the first carpometacarpal (CMC) joint has been successfully managed with various surgical treatments including trapeziectomy alone, trapeziectomy and ligament reconstruction with or without tendon interposition, metacarpal osteotomy, CMC arthrodesis, and CMC prosthetic arthroplasty; currently, no single approach has shown superiority. ${ }^{1}$

Trapeziectomy and suspensionplasty with a commercially available Mini TightRope system (Arthrex, Naples, FL) has been recently reported as an option to treat CMC degenera- tive joint disease. This system utilizes FiberWire suture secured by two stainless steel buttons in the first and second metacarpals to recreate the stabilizing force of the anterior oblique ligament and prevent first metacarpal subsidence after trapeziectomy. Cox et al and Yao and Lashgari have detailed this technique. ${ }^{2,3}$ After using guidewire placement to establish entry and exit sites in the bases of the first and second metacarpals, the Mini TightRope apparatus is inserted with accompanying suture buttons and the apparatus is tensioned. Early active range-of-motion (ROM) exercises received

November 10, 2015

accepted

November 23, 2015

published online

January 6, 2016
Copyright (c) 2016 by Thieme Medical Publishers, Inc., 333 Seventh Avenue, New York, NY 10001, USA. Tel: +1(212) 584-4662.
DOI http://dx.doi.org/ 10.1055/s-0035-1570397. ISSN 2163-3916. 
have been recommended with protection of a thumb orthosis for up to 10 weeks.

Reported benefits include decreased donor-site morbidity associated with tendon harvest and transfer, lack of need for K-wire fixation, prevention of metacarpal subsidence with immediate postoperative stability, reduced operative time, and reduced postoperative immobilization and recovery time. ${ }^{3,4}$ Although initial reported outcomes have appeared promising, long-term data on complications and safety are lacking. One case report previously reported an index metacarpal fracture after TightRope suspensionplasty, ${ }^{5}$ and another series reported two cases of a symptomatically prominent second metacarpal button and one case of postoperative infection. ${ }^{4}$ We report a complication of extensor pollicis longus (EPL) rupture after trapeziectomy and suspensionplasty using Mini TightRope suture button fixation.

\section{Case Report}

A 71-year-old right-hand-dominant female underwent right CMC trapeziectomy with Mini TightRope suspensionplasty at another center. She was immobilized in a thumb spica splint, and motion was started 2 weeks postoperatively. On her second therapy session, she noted acute-onset loss of thumb extension.

The patient moved to a new state and presented to our clinic at 3 weeks postoperatively. Examination revealed superficial wound dehiscence of $5 \mathrm{~mm}$ over the first metacarpal incision with underlying granulation tissue. There was no drainage or erythema. The patient was unable to extend the thumb interphalangeal (IP) joint. Radiographs revealed no fracture (-Fig. 1). Magnetic resonance imaging (MRI) demonstrated no abscess (-Fig. 2). We offered the patient an extensor indicis proprius (EIP) to EPL transfer.

A standard EIP to EPL tendon transfer was performed before exploring the previous first metacarpal incision to prevent contamination of a potentially infected wound. A ruptured EPL tendon slip was visualized with some surrounding pseudotendon at the level of the suture button (-Fig. $\mathbf{3}$ ). After EIP tendon transfer and wound closure, the prior incision over the first metacarpal was then explored, revealing local capsular and dermal attenuation over the suture button but no purulence. The implant was well fixed. This prior metacarpal wound was irrigated and closed. Intraoperative cultures and Gram stain were negative for infection.

At 3 months postoperatively, the patient had near-complete metacarpophalangeal and IP joint extension (- Fig. 4). At latest follow-up at 6 months postoperatively, the patient resumed normal use of her thumb.

\section{Discussion}

We report a case of EPL rupture 3 weeks after a trapeziectomy and Mini TightRope suspensionplasty, a previously unreported complication of this procedure. There are several possible reasons to account for this complication. We feel that the most likely reason for this complication was the prominent suture button causing dehiscence of the capsule and skin, allowing for the EPL tendon to desiccate and rupture. This wound dehiscence over the button may have allowed for degeneration of the tendon from exposure to air. In vitro studies have shown that exposure of biologically intact tendons to air resulted in decreased synthesis of matrix proteins, increased inflammation, and promoted fibrosis. ${ }^{6,7}$ This patient had thin skin and little subcutaneous fat, and in this particular setting a prominent subcutaneous implant may have allowed for wound breakdown close to the surrounding extensor tendons. A comparison of abductor pollicis longus suspensionplasty versus Mini TightRope suture button suspensionplasty by Avant and colleagues reported a $7 \%$ rate of symptomatically palpable suture button implants over the second metacarpal $(2 / 27$

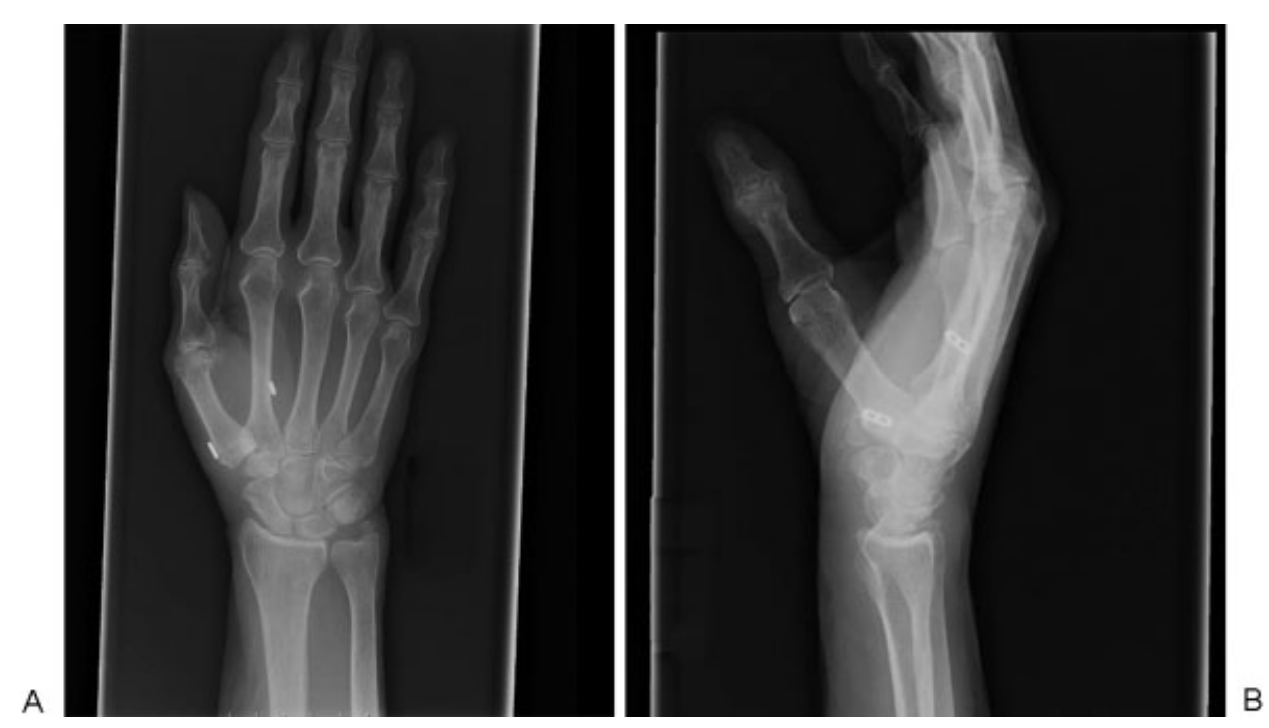

Fig. 1 Posteroanterior (A) and lateral radiographs (B) of the right wrist demonstrating a patient status post trapeziectomy with tightrope suspensionplasty and suture buttons in appropriate position. 

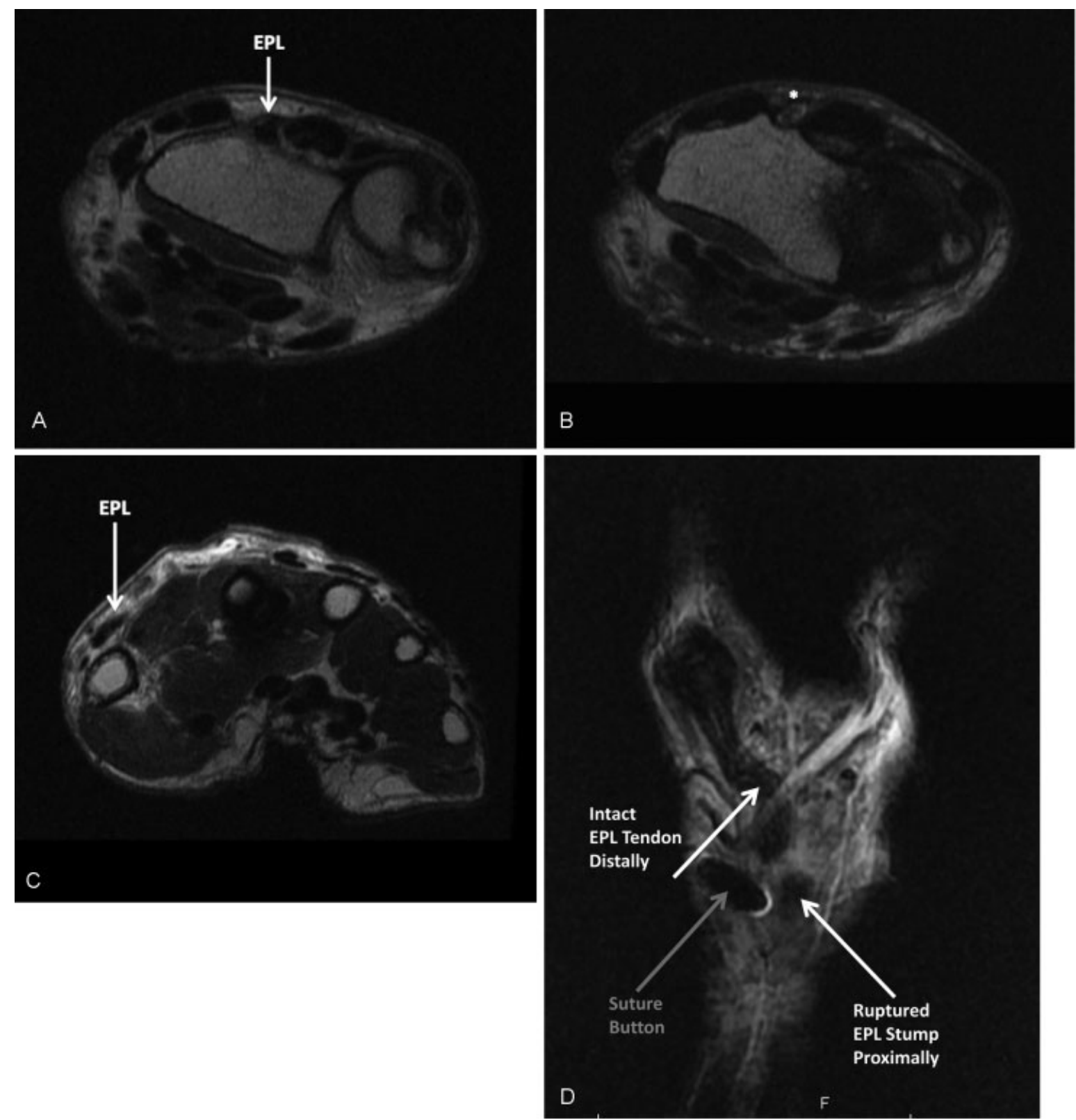

Fig. 2 Axial T2 images of the right wrist at the level of Lister's tubercle with an intact EPL tendon (A) and just distal to this point (B) revealing surrounding fluid and absence of tendon in Lister's tubercle (denoted by *). At the level of the first metacarpal midshaft (C) the EPL can be clearly seen again. Coronal STIR image (D) revealing stump of ruptured EPL tendon at metacarpal base just distal to the Mini TightRope stainless steel button.

cases), but no incidences of prominent implants in the first metacarpal. ${ }^{4}$

Another possible explanation could be tendon rupture from abrasion against the suture button implant at the base of the first metacarpal, promoted by early ROM of the tendon over the implant. EPL rupture has been documented in other situations of implant prominence, most notably in distal radius fractures treated with volar locking plates in which excessively long distal locking screws are prominent in proximity to Lister tubercle. ${ }^{8}$ At the time of surgical exploration in the current case, the ruptured distal end of the EPL tendon was in close proximity to the button, and this was also verified on preoperative MRI imaging (-Fig. 2D). Cox and colleagues recommended that the suture button be placed just ulnar to the radial aspect of the abductor pollicis brevis muscle belly and away from the more ulnar EPL tendon in the third compartment. ${ }^{2}$ Improper seating of the suture button over the line of pull of the EPL tendon may have resulted in direct abrasion of the tendon and eventual rupture. Preoperative radiographs and intraoperative inspection, however, revealed that the button was slightly radial to the line of pull of the native EPL tendon, making this explanation less likely.

Lastly, a less likely possible explanation for EPL rupture is the initiation of an acute inflammatory reaction to the suture material. FiberWire suture, which has a unique ultra-highmolecular-weight polyethylene monofilament core, has been shown to rarely incite soft tissue reaction, and case reports have shown soft tissue irritation and granuloma formation 


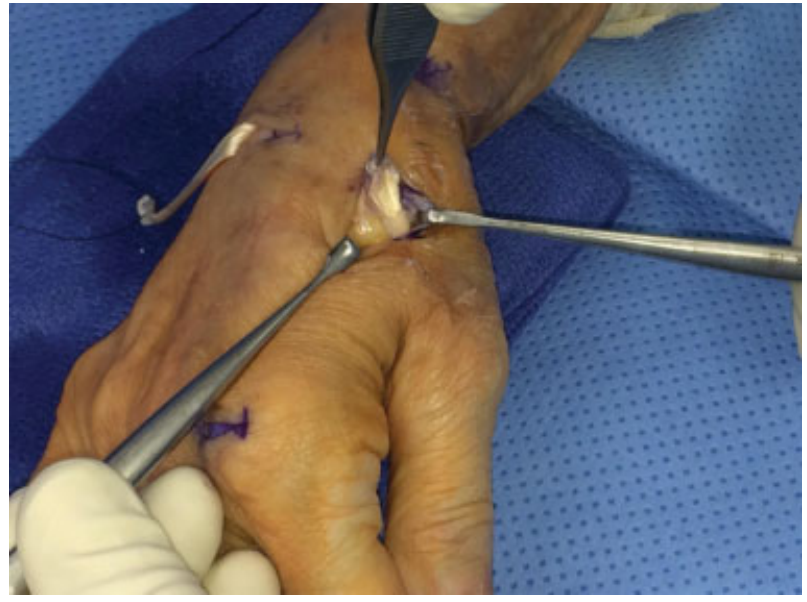

Fig. 3 Intraoperative photographs of the right hand revealing EPL rupture.

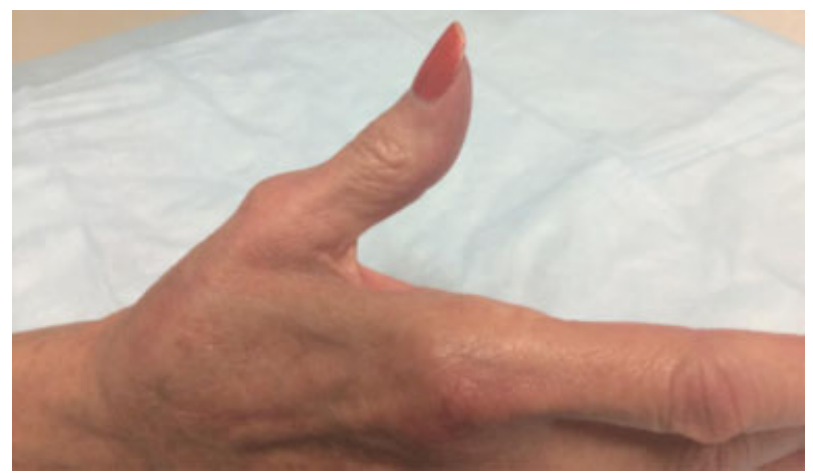

Fig. 4 Clinical photograph of the appearance of the affected right hand at 3 months postoperatively.

mimicking infection and causing a severe soft tissue inflammatory response. ${ }^{9,10}$ Previous reports have hypothesized that small particle polyethylene suture debris may promote macrophage activity and an associated inflammatory response, findings which have been demonstrated on postoperative histological analysis.

This patient's EIP to EPL tendon transfer successfully restored thumb extension (-Fig. 4). An additional benefit of the EIP transfer in this particular setting is that it offers a slightly more ulnar vector of pull of the transferred EIP, thus potentially placing the transferred tendon further from the site of the suture button on the dorsal radial base of the first metacarpal.
The Arthrex Mini TightRope apparatus has been reported to offer advantages including decreased operating time, less donor-site morbidity, decreased metacarpal subsidence, and decreased immobilization and recovery times. ${ }^{3,4}$ As its utilization increases in the treatment of CMC disease, complications such as documented in this case report will need to be further studied.

\section{Funding \\ None.}

\section{Conflict of Interest}

None.

\section{References}

1 Haase SC, Chung KC. An evidence-based approach to treating thumb carpometacarpal joint arthritis. Plast Reconstr Surg 2011;127(2):918-925

2 Cox CA, Zlotolow DA, Yao J. Suture button suspensionplasty after arthroscopic hemitrapeziectomy for treatment of thumb carpometacarpal arthritis. Arthroscopy 2010;26(10):1395-1403

3 Yao J, Lashgari D. Thumb basal joint: utilizing new technology for the treatment of a common problem. J Hand Ther 2014;27(2): 127-132, quiz 133

4 Avant KR, Nydick JA, White BD, Vaccaro L, Hess AV, Stone JD. Basal joint osteoarthritis of the thumb: comparison of suture button versus abductor pollicis longus suspensionplasty. Hand (NY) 2015;10(1):80-84

5 Khalid M, Jones ML. Index metacarpal fracture after tightrope suspension following trapeziectomy: case report. J Hand Surg Am 2012;37(3):418-422

6 Abrahamsson SO, Lohmander LS, Lundborg G. Dehydration inhibits matrix synthesis and cell proliferation. An in vitro study of rabbit flexor tendons. Acta Orthop Scand 1991;62(2):159-162

7 Saygi B, Yildirim Y, Cabukoğlu C, Kara H, Ramadan SS, Esemenli T. The effect of dehydration and irrigation on the healing of Achilles tendon: an experimental study. Ulus Travma Acil Cerrahi Derg 2008;14(2):103-109

8 Zenke Y, Sakai A, Oshige T, et al. Extensor pollicis longus tendon ruptures after the use of volar locking plates for distal radius fractures. Hand Surg 2013;18(2):169-173

9 Ollivere BJ, Bosman HA, Bearcroft PW, Robinson AH. Foreign body granulomatous reaction associated with polyethelene 'Fiberwire (®)' suture material used in Achilles tendon repair. Foot Ankle Surg 2014;20(2):e27-e29

10 Willmott HJ, Singh B, David LA. Outcome and complications of treatment of ankle diastasis with tightrope fixation. Injury 2009; 40(11):1204-1206 